\title{
Ultrastructure and molecular cytogenetics of human metaphase II oocytes with granular vacuoles
}

Célia Soares", Ana Raquel Azevedo ${ }^{* * *}$, Carolina Almeida ${ }^{* * *}$, Elsa Oliveira ${ }^{*}$, Ângela Alves ${ }^{*}$, Joaquina Silva $^{* *}$, Rosália Sá, Alberto Barros ${ }^{* * * * *}$ and Mário Sousa ${ }^{* * *, * * *}$

*Laboratory of Cell Biology, Institute of Biomedical Sciences Abel Salazar of University of Porto, Lg Prof Abel Salazar 2, 4009-003 Porto, Portugal

**Centre for Reproductive Genetics Alberto Barros, Av. do Bessa $5911^{\circ}$ Dto. Frente, 4100-009

Porto, Portugal

***Department of Genetics, Faculty of Medicine of University of Porto, Alameda Prof. Hêrnani

Monteiro, 4200-319 Porto, Portugal

celia.cmsoares@gmail.com

Studies demonstrated poor fertilization rates and compromised early embryo development in cases of oocytes with dark and granular ooplasm, or with central granular ooplasm and presence of a refractile body [1]. Many other structural abnormalities of human mature oocytes do exist, but remain to be evaluated.

In the present study we characterized granular vacuoles (GVa) of mature metaphase II human oocytes.

Surplus abnormal MII oocytes ( $n=6)$ with GVa were used under informed consent, after controlled superovulation during IVF treatment cycles. In all cases, patients had normal karyotypes. For electron microscopy oocytes were fixed with karnovsky fixative for $2 \mathrm{~h}$ at $4^{\circ} \mathrm{C}$, post-fixed in $1-2 \%$ $\mathrm{OsO}_{4}$ in buffer containing $0,8 \% \mathrm{~K}_{3} \mathrm{Fe}^{3+}(\mathrm{CN})_{6}$, dehydrated in ethanol series, equilibrated with propylene oxide and embedded in Epon. Semithin sections were stained with aqueous azur II and methylene blue (1:1). Ultrathin sections were stained with $3 \%$ aquous uranyl acetate (20 min) and Reynols lead citrate $(10 \mathrm{~min})$. Sections were observed with a transmission electron microscope JEOL 100CXII operated at $60 \mathrm{kV}$.

For cytogenetic analysis (FISH) were used four probes: for centromeric region of chromosome $\mathrm{X}$ (CEP X spectrum green: 0,1 $\mu \mathrm{l}$ ); heterochromatin region of chromosome Y (Y SAT III 0,5 $\mu \mathrm{l}$ spectrum red $+0,5 \mu \mathrm{l}$ spectrum green); centromeric region of chromosome 18 (CEP 18 spectrum blue: $1 \mu \mathrm{l}$ ) and for telomeric region of chromosome 21 (Lsi 21 spectrum red: $1 \mu \mathrm{l}$ ). Slides were mounted with $10 \mu \mathrm{l}$ of Vectashield antifade medium (Vector Laboratories, Burlingame, CA, USA) containing 1,5 $\mu \mathrm{g} / \mathrm{ml}$ of 4',6-diamidino-2-phenylindole (DAPI) to counterstain the DNA. Images were recorded using Carl Zeiss Inc. (Axioimager Z1) epifluorescence microscope.

The oocytes showed a few submembranar cortical vesicles, smooth endoplasmic reticulum (SER) tubular aggregates (T) and SER small vesicles, a total absence of SER large vesicles, and an ooplasm enriched with isolated SER cisternae. This indicates developmental immaturity of the SER, which was unable to build the vesicular-mitochondria complexes needed for fertilization. GVa were found to correspond to micronuclei, with a double membrane, chromatin and nucleolus, and inclusion of lipid droplets (Figs.1-4). This incorporation of lipid droplets were responsible for the granulated appearance of the vacuoles. They also conferred the appearance of lipofuscin bodies, thus suggesting that GVa might be in the process of autophagy. FISH analysis revealed the presence of chromatin with an aneuploid signal, thus confirming GVa are micronuclei, despite the 
presence of a metaphase II plate (Figs.5-7). The data suggest that MII oocytes with GVa should not be used for IVF due to the existence of aneuploidy.

\section{References}

[1]. Ebner et al. Hum Reprod Update 9 (2003) 251-62.

[2]. Alves et al. Eur J Hum Genet 10 (2002) 467.

\section{Acknowledgments: FCT (SFRH/BD/23616/2005; POCI/SAU-MMO/60709/60555/59997/04; UMIB}

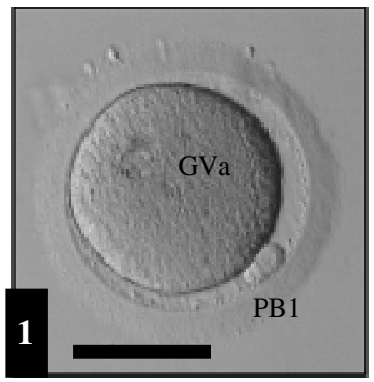

Fig.1. Inverted microscopy of a MII oocyte with granular vacuoles (GVa) in the opposite side of the first polar body (PB1). Bar=10 $\mu \mathrm{m}$.

Fig.2. Cortex of oocyte had a reduced number of cortical vesicles (cv), smooth endoplasmatic reticulum (SER), tubular aggregates (T), small (sv) and medium (mv) size vesicles. SER large vesicles were completely absent. The ooplasm was enriched in SER isolated tubules (t). Mitochondria (m); Zona pellucida $(\mathrm{ZP})$. Bar $=2,5 \mu \mathrm{m}$.
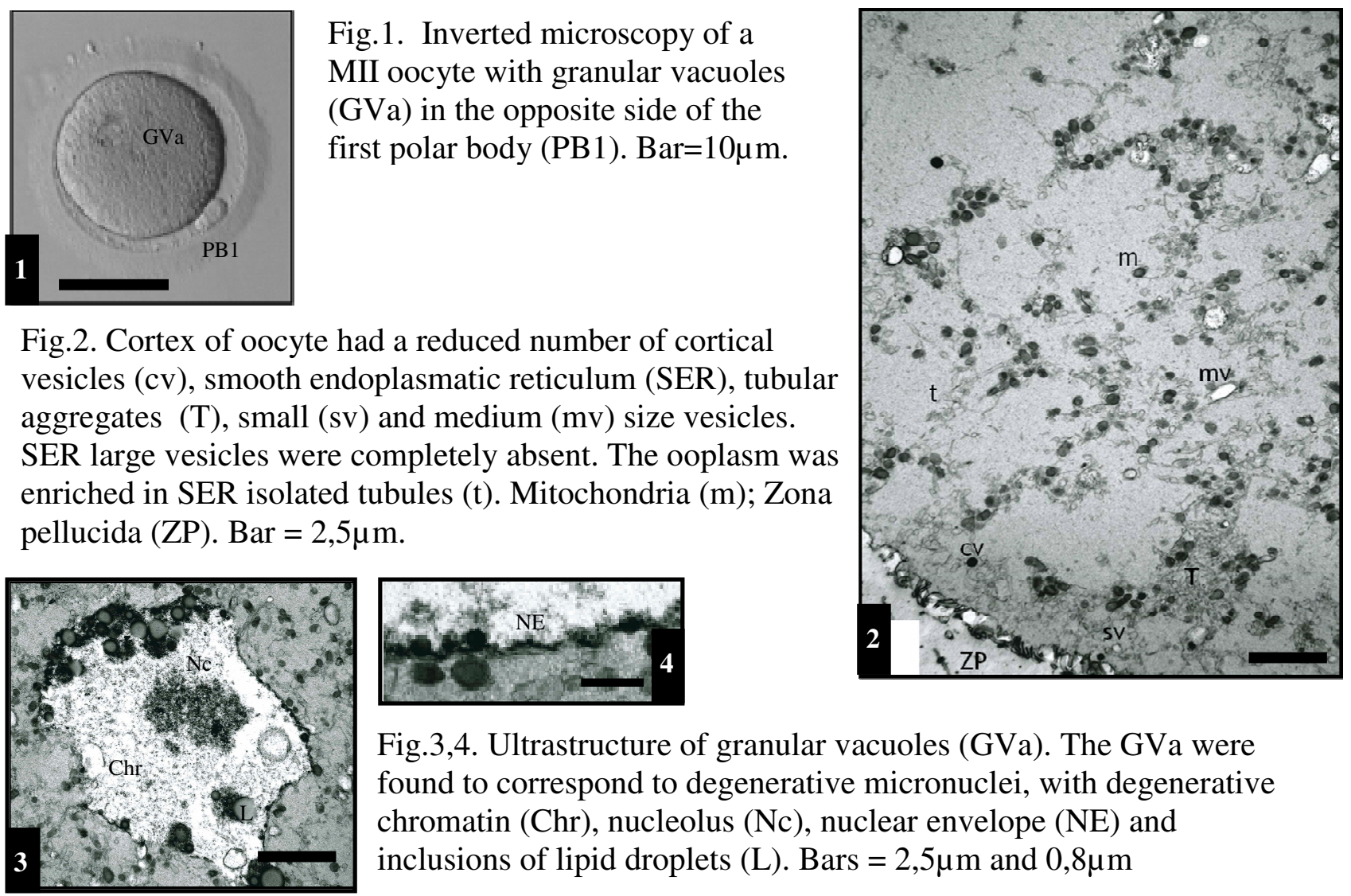

Fig.3,4. Ultrastructure of granular vacuoles (GVa). The GVa were found to correspond to degenerative micronuclei, with degenerative chromatin (Chr), nucleolus (Nc), nuclear envelope (NE) and inclusions of lipid droplets $(\mathrm{L})$. Bars $=2,5 \mu \mathrm{m}$ and $0,8 \mu \mathrm{m}$
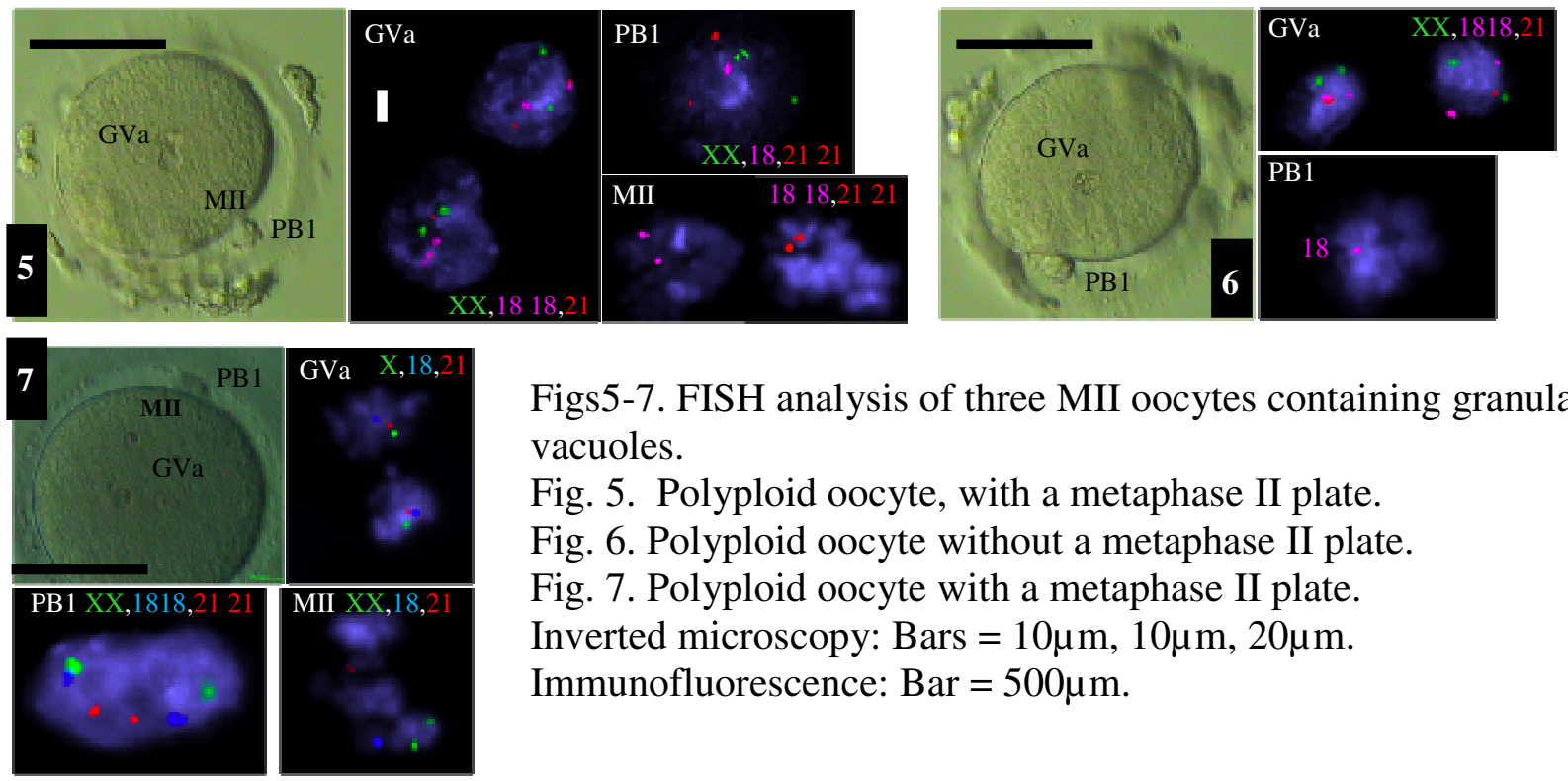

Figs5-7. FISH analysis of three MII oocytes containing granular vacuoles.

Fig. 5. Polyploid oocyte, with a metaphase II plate.

Fig. 6. Polyploid oocyte without a metaphase II plate.

Fig. 7. Polyploid oocyte with a metaphase II plate.

Inverted microscopy: Bars $=10 \mu \mathrm{m}, 10 \mu \mathrm{m}, 20 \mu \mathrm{m}$.

Immunofluorescence: $\mathrm{Bar}=500 \mu \mathrm{m}$. 